\title{
Asymmetrically Clipped Absolute Value Optical OFDM for Intensity-Modulated Direct-Detection Systems
}

\author{
Ruowen Bai, Student Member, IEEE, Qi Wang, Member, IEEE, Zhaocheng Wang, Senior Member, IEEE
}

\begin{abstract}
Orthogonal frequency division multiplexing (OFD$M)$ is attracting increasing attention in optical communication systems thanks to the inherent benefits such as high spectral efficiency and resistance to frequency-selective channels. In this paper, a novel energy and spectrally efficient scheme called asymmetrically clipped absolute value optical OFDM (AAOOFDM) is proposed for intensity-modulated direct-detection (IM/DD) systems. In AAO-OFDM, absolute value optical OFDM (AVO-OFDM) signals on the even subcarriers and asymmetrically clipped optical OFDM (ACO-OFDM) signals on the odd subcarriers are combined for simultaneous transmission, which employs all the subcarriers requiring no DC biases. For AVOOFDM scheme, the frequency symbols are firstly modulated on the even subcarriers, which are then fed into an inverse fast Fourier transform block. Afterwards, the absolute values of the bipolar time-domain signals are taken to guarantee nonnegativity, while their signs are mapped to the complex-valued symbols and modulated on the odd subcarriers. Since there remain unused odd subcarriers, other useful symbols can be modulated on them, which leads to the conventional ACO-OFDM scheme. At the receiver, the ACO-OFDM symbols on the odd subcarriers are demodulated firstly, which are reconstructed and removed from the received signals. Afterwards, the remaining signals are utilized to detect the AVO-OFDM symbols with the aid of the demodulated sign symbols on the odd subcarriers. Theoretical analysis and simulation results show that AAO-OFDM has lower peak-to-average power ratio than other optical OFDM schemes, which makes it less sensitive to the nonlinearity of the optical devices. Furthermore, it achieves better bit error rate performance compared to its counterparts for the same spectral efficiency.
\end{abstract}

Index Terms-Intensity modulation with direct detection, orthogonal frequency division multiplexing, energy efficiency, spectral efficiency.

This work was supported in part by National Key Basic Research Program of China under Grant 2013CB329203, in part by Shenzhen Subject Arrangements (JCYJ20160331184124954), in part by Shenzhen Peacock Plan (No. 1108170036003286), in part by Shenzhen Fundamental Research Project (JCYJ20150401112337177), and in part by EPSRC funded projects (Grant Nos. EP/N004558/1 and EP/N023862/1). The data from the paper can be obtained from the University of Southampton Pure research repository: http://doi.org/10.5258/SOTON/D0123. (Corresponding author: Zhaocheng Wang.)

R. Bai, and Z. Wang are with the Tsinghua National Laboratory for Information Science and Technology (TNList), Department of Electronic Engineering, Tsinghua University, Beijing 100084, China (e-mail: brw15@mails.tsinghua.edu.cn; zcwang@tsinghua.edu.cn).

Q. Wang is with the School of Electronics and Computer Science, University of Southampton, Southampton SO17 1BJ, U.K. (e-mail: qw1e16@soton.ac.uk).

Copyright (c) 2017 IEEE. Personal use of this material is permitted. However, permission to use this material for any other purposes must be obtained from the IEEE by sending a request to pubs-permissions@ieee.org.

\section{INTRODUCTION}

Intensity-modulated direct-detection (IM/DD) systems are widely employed in optical wireless communication (OWC) [1]-[3], fiber-optic communication [4], amplitude modulated radio frequency (RF) wireless communication [5], and broadband wireline transmission [6]. For a general IM/DD system, the instantaneous output optical power is proportional to the input electrical current and the data information is modulated onto the input current intensity. The input electrical current signal has to be real-valued and nonnegative [1]-[8]. At the receiver, to convert the optical signal to electrical signal, direct detection is employed, in which a photo-diode (PD) is used to produce a current proportional to the received instantaneous optical power. In this paper, an equivalent linear time-invariant (LTI) channel is assumed, and the optical channel nonlinearity is not considered since several techniques have been proposed to mitigate the nonlinearity effect [9]-[11]. In general, this paper relates to the digital domain signals only.

Orthogonal frequency division multiplexing (OFDM) is one of the popular physical layer schemes utilized in a wide range of RF communications, and it is also considered as a candidate for IM/DD optical systems, since OFDM has inherent benefits such as high spectral efficiency, resistance to frequency-selective channels and simple one-tap equalization [12]-[14]. Hermitian symmetry is commonly imposed on the input data of the OFDM block to generate real-valued timedomain output signals [14]. Some optical OFDM schemes are proposed to make the bipolar output signals nonnegative, such as DC biased optical OFDM (DCO-OFDM) and asymmetrically clipped optical OFDM (ACO-OFDM). In DCO-OFDM, a DC bias is added to the bipolar real-valued time-domain signals, and all the remaining negative signals are clipped at zero. The clipping operation will cause clipping distortion, which limits its performance. An appropriate DC bias should vary with the constellation sizes to ensure good performance, which makes the transmitter complex. In addition, the DC bias consumes high power and reduces the energy efficiency of DCO-OFDM. To improve the energy efficiency, ACO-OFDM is proposed, where only the odd subcarriers are utilized and the time-domain signals have antisymmetry property [15]-[17]. Hence the negative parts can be clipped without losing any information and its clipping distortion only falls on the even subcarriers. However, since only half of the subcarriers are employed, ACO-OFDM is spectrally inefficient.

Other optical OFDM schemes have been proposed to over- 
come the drawbacks of DCO-OFDM and ACO-OFDM, such as asymmetrically clipped DC-biased optical OFDM (ADOOFDM) [18], [19], hybrid asymmetrically clipped optical OFDM (HACO-OFDM) [14], asymmetrically and symmetrically clipped optical OFDM (ASCO-OFDM) [20], etc. In ADO-OFDM, ACO-OFDM symbols are modulated on the odd subcarriers while DCO-OFDM symbols are modulated on the even subcarriers. The spectral efficiency of ADO-OFDM is improved compared to ACO-OFDM, and the required DC bias is also reduced compared to DCO-OFDM when the constellation sizes are chosen appropriately. However, the DC bias is still required. In HACO-OFDM, ACO-OFDM symbols are modulated on the odd subcarriers and pulse amplitude modulated discrete multitone (PAM-DMT) symbols are modulated on the imaginary part of the even subcarriers, where the bipolar PAM-DMT time-domain signals are clipped at zero and the clipping distortion falls on the real part of the corresponding subcarriers [14], [21]. Note that the real part of the even subcarriers is wasted. As for ASCO-OFDM, ACOOFDM symbols are modulated on the odd subcarriers while symmetrically clipping optical OFDM (SCO-OFDM) symbols are modulated on the even subcarriers of two adjacent SCOOFDM blocks [20]. Since the information carried by the two adjacent SCO-OFDM blocks is from the same OFDM block, the half of the even subcarriers are wasted. Therefore, both HACO-OFDM and ASCO-OFDM are not spectrally efficient.

To further enhance the energy and spectral efficiency, asymmetrically clipped absolute value optical OFDM (AAOOFDM) is proposed in this paper. In AAO-OFDM, absolute value optical OFDM (AVO-OFDM) signals on the even subcarriers and ACO-OFDM signals on the odd subcarrier$\mathrm{s}$ are transmitted together. AVO-OFDM symbols are firstly modulated on the even subcarriers, then fed into an inverse fast Fourier transform (IFFT) block, after that the absolute values of the bipolar output signals are taken to make the signals nonnegative, and their signs are mapped to complexvalued symbols which are modulated on the odd subcarriers. Note that some odd subcarriers remains unused, which can be employed to modulate other useful symbols. The odd subcarriers modulating the sign symbols and other useful symbols employ conventional ACO-OFDM scheme. At the receiver, the ACO-OFDM symbols on the odd subcarriers are demodulated firstly, which are reconstructed and removed from the received signals. We prove that the Fourier transform of the AVOOFDM time-domain signals are zeros on the odd subcarriers, thus the performance of ACO-OFDM is not degraded by AVOOFDM. Since the clipping distortion of ACO-OFDM also falls on the even subcarriers only, we can recover the ACOOFDM symbols by demodulating the odd subcarriers using conventional ACO-OFDM receiver. Afterwards, we can regenerate local estimation of ACO-OFDM time-domain clipping distortion, which is removed from the received signals. After that, the remaining signals are used to detect the AVO-OFDM symbols with the aid of the demodulated sign symbols.

Compared to the conventional optical OFDM schemes, AAO-OFDM has various advantages. Firstly, no DC bias is required at the transmitter, hence it is more energy efficient than DCO-OFDM and ADO-OFDM. In addition, since both the real and imaginary parts of the even and odd subcarriers are utilized, AAO-OFDM is more spectrally efficient than ACO-OFDM, HACO-OFDM and ASCO-OFDM. Moreover, ACO-OFDM and AVO-OFDM in AAO-OFDM have similar performance when the signal to noise ratio (SNR) is relatively high and the same constellation sizes are used, which is preferred in practical communication systems. Furthermore, the peak-to-average power ratio (PAPR) of AAO-OFDM is lower than that of ACO-OFDM, ASCO-OFDM and HACOOFDM, which makes it less sensitive to the nonlinearity of the optical devices. Therefore, AAO-OFDM is a more attractive modulation scheme for IM/DD optical systems compared to other optical OFDM schemes.

The remainder of this paper is organized as follows. In Section II, the overview of optical OFDM is presented, while in Section III, the proposed AAO-OFDM and its transceiver are developed. In Section IV, the probability density function (PDF) and the PAPR of AAO-OFDM time-domain signals are analyzed, and the spectral efficiency, optical power allocation and computational complexity analysis are provided. In Section $\mathrm{V}$, the bit error ratio (BER) performance of AAO-OFDM is simulated and compared to other optical OFDM schemes, while the conclusions are drawn in Section VI.

\section{OVERVIEW OF OPTICAL OFDM}

In this section, the conventional optical OFDM schemes including ACO-OFDM, DCO-OFDM and ADO-OFDM are briefly summarized.

\section{A. DCO-OFDM}

In a DCO-OFDM based IM/DD system with $N$ subcarriers, where $N$ is typically a large even number, all the subcarriers carry useful symbols except that $X_{0}$ and $X_{N / 2}$ are set to zeros, and thus the complex-valued frequency-domain symbols are given by

$$
\mathbf{X}=\left[0, X_{1}, X_{2}, \cdots, X_{N / 2-1}, 0, X_{N / 2-1}^{*} \cdots, X_{2}^{*}, X_{1}^{*}\right],
$$

where Hermitian symmetry is constrained to make the timedomain signals real-valued, and we have $X_{k}=X_{N-k}^{*}, 1 \leq$ $k \leq N / 2-1$.

After an IFFT block, the time-domain signals $\left\{x_{n}\right\}$ are obtained, which are given by

$$
x_{n}=\frac{1}{\sqrt{N}} \sum_{k=0}^{N-1} X_{k} \exp \left(j \frac{2 \pi}{N} n k\right), 0 \leq n \leq N-1 .
$$

After that, a DC bias is added and all the remaining negative peaks are clipped, leading to nonnegative DCOOFDM time-domain signals $\left\{x_{\mathrm{dco}, n}\right\}$. The DC bias level can be set according to the standard deviation $\sigma_{x}=\sqrt{E\left\{x_{n}^{2}\right\}}$, which is given by [19], [22]

$$
B_{\mathrm{DC}}=k \sigma_{x},
$$

where $k$ is a non-negative proportionality constant. As in [22], the bias-index can be defined as $\beta=10 \log _{10}\left(1+k^{2}\right)[\mathrm{dB}]$, which expresses the power ratio between the DC-biased signal to the non-DC-biased signal. A small DC bias causes 
severe clipping distortion and a high DC bias causes energy inefficiency. Thus, an appropriate DC bias, which balances the clipping distortion and the energy inefficiency, is studied to enhance the DCO-OFDM performance in some literatures [23]-[25].

\section{B. ACO-OFDM}

In an ACO-OFDM based IM/DD system, only the odd subcarriers carry useful symbols, while all the even subcarriers are set to zeros. To guarantee that the time-domain signals are realvalued, Hermitian symmetry is imposed on the $N$ subcarriers. Therefore, the frequency-domain symbols of ACO-OFDM are given by [15]-[17]

$$
\mathbf{Y}=\left[0, Y_{1}, 0, Y_{3}, \cdots, Y_{N / 2-1}, 0, Y_{N / 2-1}^{*}, \cdots, Y_{1}^{*}\right] .
$$

After the process of IFFT, the time-domain signals $\left\{y_{n}\right\}$ are obtained, which have an antisymmetry property as [15]-[17]

$$
y_{n}=-y_{n+N / 2}, \quad 0 \leq n \leq N / 2-1 .
$$

Therefore, we can clip the negative part of the time-domain signals without losing any information as

$$
y_{\text {aco }, n}=\frac{1}{2}\left(y_{n}+c_{\text {aco }, n}\right), 0 \leq n \leq N-1,
$$

where $c_{\mathrm{aco}, n}$ denotes the clipping distortion of ACO-OFDM, which is given by

$$
c_{\mathrm{aco}, n}=\left|y_{n}\right|,
$$

where $|\cdot|$ denotes an absolute value operator.

Since the Fourier transform of the clipping distortion only falls on the even subcarriers [15]-[17], the ACO-OFDM symbols can be directly detected on the odd subcarriers after the received signals are fed to an $N$-point FFT block at the receiver.

\section{ADO-OFDM}

ADO-OFDM is developed in [18], [19], in which ACOOFDM symbols are modulated on the odd subcarriers while DCO-OFDM symbols are modulated on the even subcarriers. All the negative values generated by ACO-OFDM and DCOOFDM are clipped separately. After that, the sum of the remaining nonnegative signals are transmitted together.

For a typical IM/DD system using ADO-OFDM with $N$ subcarriers, the complex-valued symbols on the even subcarriers are given by

$$
\mathbf{X}=\left[0,0, X_{2}, 0, \cdots, X_{N / 2-2}, 0,0,0, X_{N / 2-2}^{*}, \cdots, X_{2}^{*}, 0\right],
$$

where Hermitian symmetry is employed on the $N$ subcarriers.

$\mathbf{X}$ symbol vector is converted by an $N$-point IFFT to generate the time-domain signals $\left\{x_{n}\right\}$. After that, a DC bias is added and all the remaining negative peaks are clipped, leading to nonnegative signals $\left\{x_{\mathrm{dco}, n}\right\}$. The DC bias can be set according to (3).

In addition, the symbols on the odd subcarriers are given as (4). Similarly, $\mathbf{Y}$ symbol vector is converted by an $N$ point IFFT to yield the time-domain signals $\left\{y_{n}\right\}$, which have antisymmetry property as (5). Thus ACO-OFDM time-domain signals $\left\{y_{\text {aco }, n}\right\}$ are obtained through clipping the negative part of $\left\{y_{n}\right\}$ without losing any information.

At the receiver, the thermal noise and shot noise are modeled as additive white Gaussian noise (AWGN) [16]. Therefore, the received signals are given by

$$
r_{\text {ado }, n}=h_{n} \otimes\left(x_{\mathrm{dco}, n}+y_{\text {aco }, n}\right)+w_{n}, 0 \leq n \leq N-1,
$$

where $h_{n}$ and $w_{n}$ are the sample of channel state information and AWGN, respectively, and $\otimes$ denotes the convolution operator. The symbols on the odd subcarriers are demodulated by conventional ACO-OFDM receiver and the symbols on the even subcarriers are demodulated after interference cancelation process [19].

\section{PROPOSED AAO-OFDM}

In this section, we present the transceiver design of the AAO-OFDM. In AAO-OFDM, AVO-OFDM signals on the even subcarriers and ACO-OFDM signals on the odd subcarriers are transmitted together. The AVO-OFDM scheme is also proposed in this paper, where the optical OFDM symbols are firstly modulated on the even subcarriers, then fed into an IFFT block leading to bipolar time-domain signals. After parallel to serial (P/S) converter, ideal digital to analog (D/A) converter and low pass filter (LPF), the absolute values of the bipolar time-domain signals are taken to guarantee the nonnegativity, while their sign information is mapped to the complex-valued symbols and modulated on the odd subcarriers. Since there remain unused odd subcarriers, other useful symbols can be modulated on them, which leads to the conventional ACOOFDM scheme.

\section{A. AAO-OFDM Transmitter}

For an AAO-OFDM based IM/DD system with $N$ subcarriers, the block diagram of the transmitter is shown in Fig. 1. To generate real-valued time-domain signals, Hermitian symmetry is imposed on the $N$ subcarriers, where we have $X_{k}=X_{N-k}^{*} . X_{0}$ and $X_{N / 2}$ are set to zero. Then AVO-OFDM frequency-domain symbol vector $\mathbf{X}$ is given by

$$
\mathbf{X}=\left[0,0, X_{2}, 0, \cdots, X_{N / 2-2}, 0,0,0, X_{N / 2-2}^{*}, \cdots, X_{2}^{*}, 0\right],
$$

where $X_{k}\left(2 \leq k \leq \frac{N}{2}-2\right)$ is the complex-valued symbol on the $k$ th subcarrier, according to the chosen constellations, such as pulse amplitude modulation (PAM), phase shift keying (PSK), and quadrature amplitude modulation (QAM), etc. Without loss of generality, the QAM constellation is assumed for AVO-OFDM symbols and its size is set to be $M_{1}$.

According to (2), $\mathbf{X}$ symbol vector is converted by an $N$ point IFFT block to yield the time-domain signal vector $\mathbf{x}$, which has a symmetry property, as [18], [19]

$$
x_{n}=x_{n+N / 2}, 0 \leq n \leq N / 2-1 .
$$

Then the signal vector $\mathbf{x}$ is converted from parallel to serial, and the resulting signal is D/A converted and low-pass filtered, after which the absolute operation is employed to obtain 


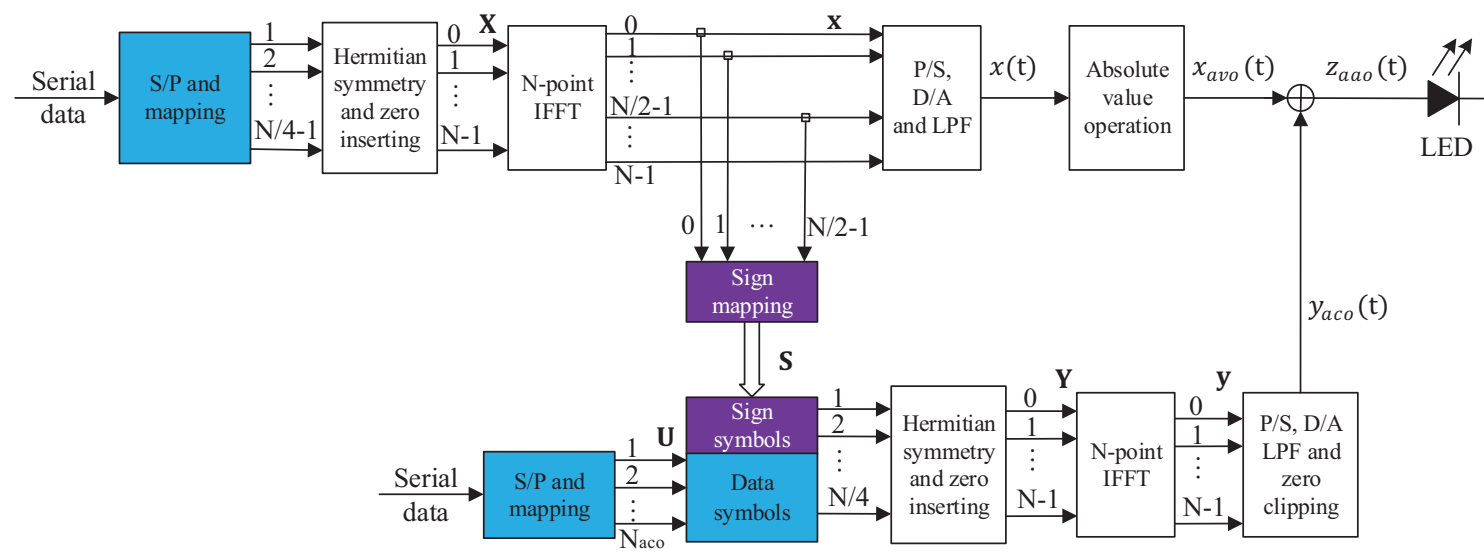

Fig. 1. The block diagram of AAO-OFDM transmitter.

$x_{\text {avo }}(t)$ as in Fig. 1. In this paper, ideal digital to analog converter (DAC) and ideal low pass filter (LPF) are assumed.

According to (11), only $N / 2$ signs of $\left\{x_{n}\right\}$ are effective, and the effective signs of $\left\{x_{n}\right\}$ are taken and mapped to $\left\{s_{n}\right\}$, given by

$$
s_{n}= \begin{cases}0, & x_{n} \leq 0 \\ 1, & x_{n}>0\end{cases}
$$

where $0 \leq n \leq N / 2-1$.

After that, $\left\{s_{n}\right\}$ are mapped to complex-valued symbols $\left\{S_{k}\right\}$ according to the chosen constellation. Without loss of generality, the QAM constellation is assumed and its size is set to $M_{\text {sign }}$. The number of the sign symbols $S_{k}$ is assumed to be $N_{\text {sign }}$, which is given by

$$
N_{\text {sign }}=\left\lceil\frac{N / 2}{\log _{2} M_{\text {sign }}}\right\rceil,
$$

where $\lceil\zeta\rceil$ denotes the function that maps the smallest integer greater than or equal to $\zeta$. There are only $N / 4$ effective complex-valued symbols on the odd subcarriers in one frame, hence $N_{\text {sign }} \leq N / 4$ should be satisfied, and further, $M_{\text {sign }} \geq 4$ is required.

In addition, the remaining odd subcarriers can be utilized to modulate other useful complex-valued symbols $U_{k}$ according to the chosen constellation, which is assumed to be QAM and its size is set to $M_{2}$. The number of these useful symbols is assumed to be $N_{\text {aco }}$, which is given by

$$
N_{\text {aco }}=N / 4-N_{\text {sign }} .
$$

Thus, after Hermitian symmetry is imposed, the complexvalued symbol vector $\mathbf{Y}$ modulated on the odd subcarriers is given by

$$
\mathbf{Y}=\left[0, Y_{1}, 0, Y_{3}, \cdots, Y_{N / 2-1}, 0, Y_{N / 2-1}^{*}, \cdots, Y_{1}^{*}\right],
$$

where

$$
Y_{k}=\left\{\begin{array}{lc}
S_{k}, & k=1,3, \cdots, 2 N_{\text {sign }}-1 ; \\
U_{k}, & k=2 N_{\text {sign }}+1,2 N_{\text {sign }}+3, \cdots, N / 2-1 .
\end{array}\right.
$$

Similarly, $\mathbf{Y}$ symbol vector is converted by an $N$-point IFFT to yield the time-domain signal vector $\mathbf{y}$, which has an antisymmetry property given by [16], [17]

$$
y_{n}=-y_{n+N / 2}, 0 \leq n \leq N / 2-1 .
$$

Thus we can clip the negative part of $\left\{y_{n}\right\}$ without losing any information and discrete time-domain signals $\left\{y_{\text {aco }, n}\right\}$ are obtained, which are given by [19]

$$
y_{\text {aco }, n}=\frac{1}{2}\left(y_{n}+c_{\text {aco }, n}\right), 0 \leq n \leq N-1,
$$

where $c_{\mathrm{aco}, n}$ denotes the clipping distortion as in (7). After the $\mathrm{P} / \mathrm{S}, \mathrm{D} / \mathrm{A}, \mathrm{LPF}$ and zero clipping, the continuous time-domain analog signal $y_{\text {aco }}(t)$ is obtained.

As shown in Fig. 1, the AAO-OFDM time-domain signal is obtained by adding $y_{\text {aco }}(t)$ to $x_{\text {avo }}(t)$, which is given by

$$
z_{\text {aao }}(t)=x_{\text {avo }}(t)+y_{\text {aco }}(t) \text {. }
$$

Finally, the continuous time-domain analog signal $z_{\text {aao }}(t)$ is used to modulate an optical emitter. In this paper, an ideal optical emitter is assumed so that the intensity of the output optical signal is proportional to the input electrical current. The optical signal is also assumed to be transmitted across a flat channel [18], [19].

\section{B. AAO-OFDM Receiver}

The block diagram of the receiver of AAO-OFDM is shown in Fig. 2. At the receiver, the shot noise and thermal noise are commonly modeled as additive white Gaussian noise (AWGN) [18], [19]. The received signal is first converted from an optical signal to an electrical signal by an optical electrical converter, such as the photo-diode (PD), then after the processing of LPF, analog to digital (A/D), and serial to parallel $(\mathrm{S} / \mathrm{P})$, the discrete time-domain received signal $r_{n}$ is 


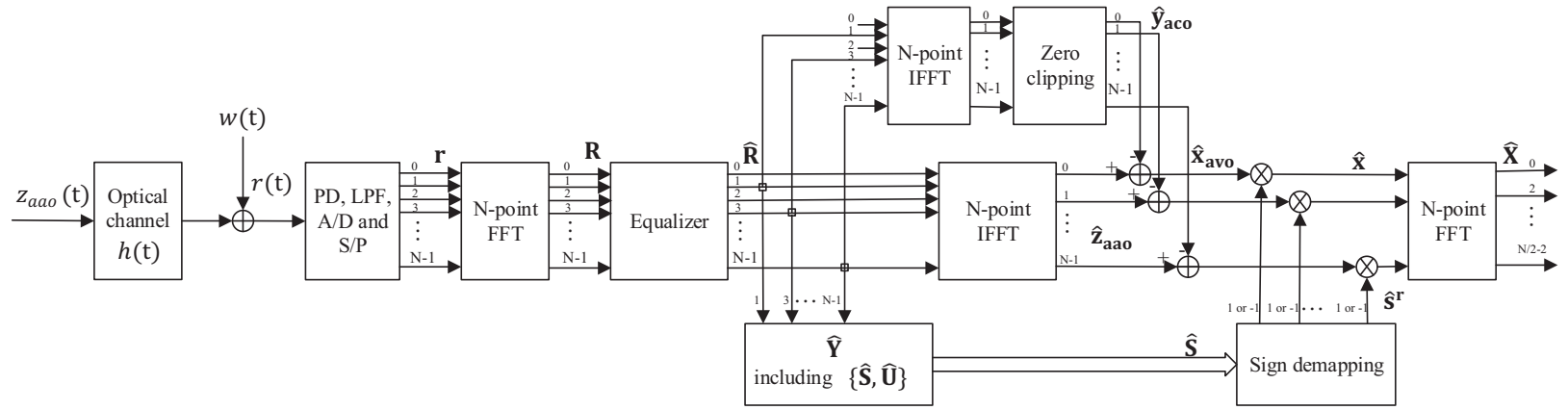

Fig. 2. The block diagram of AAO-OFDM receiver.

obtained, which is given by

$$
\begin{aligned}
& r_{n}=h_{n} \otimes z_{\mathrm{aao}, n}+w_{n} \\
& =h_{n} \otimes x_{\mathrm{avo}, n}+\frac{1}{2} h_{n} \otimes\left(y_{n}+c_{\mathrm{aco}, n}\right)+w_{n}, 0 \leq n \leq N-1,
\end{aligned}
$$

where $h_{n}$ and $w_{n}$ denote the samples of channel state information and of AWGN, respectively.

The received signals $\left\{r_{n}\right\}$ are fed to the $N$-point FFT block and the frequency-domain symbols are generated as

$$
\begin{aligned}
& R_{k}=H_{k} Z_{\text {aao }, k}+W_{k} \\
& =H_{k} X_{\text {avo }, k}+\frac{1}{2} H_{k}\left(Y_{k}+C_{\text {aco }, k}\right)+W_{k}, 0 \leq k \leq N-1,
\end{aligned}
$$

where $H_{k}, Z_{\text {aao }, k}, X_{\text {avo }, k}, C_{\text {aco }, k}$ and $W_{k}$ are the discrete Fourier transforms (DFTs) of $h_{n}, z_{\mathrm{aao}, n}, x_{\mathrm{avo}, n}, c_{\mathrm{aco}, n}$ and $w_{n}$, respectively. Since $W_{k}, 0 \leq k \leq N-1$, is the summation of $N$ independent AWGN samples $\left\{w_{n}\right\}$, it is also complex Gaussian [17].

Considering that $\left\{x_{\mathrm{avo}, n}\right\}$ have a symmetry property, we have

$$
\begin{aligned}
& X_{\mathrm{avo}, k}=\frac{1}{\sqrt{N}} \sum_{n=0}^{N-1} x_{\mathrm{avo}, n} \exp \left(-j \frac{2 \pi}{N} n k\right) \\
= & \frac{1}{\sqrt{N}} \sum_{n=0}^{N / 2-1} x_{\mathrm{avo}, n}\left\{\exp \left(-j \frac{2 \pi n k}{N}\right)\right. \\
& \left.+\exp \left(-j \frac{2 \pi\left(n+\frac{N}{2}\right) k}{N}\right)\right\} \\
= & \frac{1}{\sqrt{N}} \sum_{n=0}^{N / 2-1} x_{\mathrm{avo}, n} \exp \left(-j \frac{2 \pi}{N} n k\right)\{1+\exp (-j k \pi)\} .
\end{aligned}
$$

Thus, if $k$ is odd, then $1+\exp (-j k \pi)=0$ and $X_{\mathrm{avo}, k}=0$, which means that $X_{\mathrm{avo}, k}$ comprises the even subcarriers only and the performance of ACO-OFDM is not degraded by AVOOFDM. Note that $C_{\mathrm{aco}, k}$ only comprises the even subcarriers as well [15], [18], [19].

After $N$-point FFT and the equalizer, we could estimate the ACO-OFDM symbols on the odd subcarriers using maximum- likelihood (ML) detection, which are given by [16], [26]

$$
\begin{aligned}
& \widehat{Y}_{k}=\arg \max _{Y \in \Omega_{Y}} \operatorname{Pr}\left\{\widehat{R}_{k} \mid H_{k}, \Omega_{Y}\right\} \\
& =\arg \min _{Y \in \Omega_{Y}}\left\|H_{k} Y-2 \widehat{R}_{k}\right\|^{2}, k=1,3, \cdots, N / 2-1,
\end{aligned}
$$

where $\Omega_{Y}$ denotes the constellation set of ACO-OFDM, $\operatorname{Pr}\{A \mid B\}$ denotes the conditional probability of event $A$ given event $B, \widehat{R}_{k}$ denotes the estimation of $R_{k}$ after an equalizer as in Fig. 2, and $\|\cdot\|$ denotes the Frobenius norm. The multiplication by 2 is to compensate the clipping loss according to (21).

After that, we can obtain the estimations of the sign symbols $\left\{\widehat{S}_{k}\right\}$ and of the useful symbols $\left\{\widehat{U}_{k}\right\}$ according to (16), which are given by

$$
\widehat{S}_{k}=\widehat{Y}_{k}, k=1,3, \cdots, 2 N_{\text {sign }}-1
$$

and

$$
\widehat{U}_{k}=\widehat{Y}_{k}, k=2 N_{\text {sign }}+1,2 N_{\text {sign }}+3, \cdots, N / 2-1 .
$$

Before demodulating the AVO-OFDM symbols, we should subtract the interference caused by ACO-OFDM. As shown in Fig. 2, $\left\{\widehat{Y}_{k}\right\}$ are utilized to generate local estimation of ACOOFDM time-domain signals $\left\{\widehat{y}_{\text {aco }, n}\right\}$, which are subtracted from the received signals. After that, we can estimate the AVOOFDM time-domain symbols $\left\{\widehat{x}_{\mathrm{avo}, n}\right\}$, whose sign estimations $\left\{\widehat{s}_{n}\right\}$ could be obtained by demapping from $\left\{\widehat{S}_{k}\right\}$. According to (11), $\left\{x_{n}\right\}$ have symmetry property, hence the $N$ signs are symmetrical and the later $N / 2$ signs are equal to $\left\{\widehat{s}_{n}\right\}$ correspondingly. Thus the sign estimations of the $\left\{\widehat{x}_{n}\right\}$ at the receiver are given by

$$
\widehat{s}_{n}^{r}=\widehat{s}_{n+N / 2}^{r}=\left\{\begin{array}{cc}
1, & \widehat{s}_{n}=1 ; \\
-1, & \widehat{s}_{n}=0,
\end{array}\right.
$$

where $0 \leq n \leq N / 2-1$.

Thus, we can obtain the estimations of $\left\{x_{n}\right\}$, which are given by

$$
\widehat{x}_{n}=\widehat{s}_{n}^{r} \widehat{x}_{\mathrm{avo}, n}, n=0,1,2, \cdots, N-1 .
$$

After an $N$-point FFT block, we could obtain the estimations of $\left\{X_{k}\right\}$ on the even subcarriers using ML detection, 
which are given by

$$
\begin{aligned}
& \widehat{X}_{k}=\arg \max _{X \in \Omega_{X}} \operatorname{Pr}\left\{\widehat{X}_{k}^{r} \mid H_{k}, \Omega_{X}\right\} \\
& =\arg \min _{X \in \Omega_{X}}\left\|H_{k} X-\widehat{X}_{k}^{r}\right\|^{2}, k=2,4, \cdots, N / 2-2,
\end{aligned}
$$

where $\Omega_{X}$ denotes the constellation set of AVO-OFDM and $\widehat{X}_{k}^{r}$ denotes the DFT of $\widehat{x}_{n}$ at the receiver.

\section{Signal AnAlysis of AAO-OFDM}

In this section, the PDF and PAPR of AAO-OFDM timedomain signals, the analysis of spectral efficiency, optical power allocation and computational complexity of AAO-OFDM are presented.

\section{A. The PDF of AAO-OFDM Time-Domain Signals}

We could analyze the AAO-OFDM signals more comprehensively under the help of their PDF. Thus, the PDF is investigated in this subsection. Using the central limit theorem, it can be shown that the bipolar time-domain signals $x(t)$ and $y(t)$ follow a Gaussian distribution with zero mean [17], [27], [28], in which $x(t)$ and $y(t)$ are the output signals of the D/A for the input $x_{n}$ and $y_{n}$, respectively. This holds for large subcarrier numbers especially when $N \geq 128$ [17], [27].

Hence, after absolute value operation, the PDF of AVOOFDM time-domain signals $x_{\mathrm{avo}}(t)$ is a truncated Gaussian distribution, which is given by

$$
f_{x_{\text {avo }}(t)}(w)=\frac{2}{\sqrt{2 \pi} \sigma_{v}} \exp \left(\frac{-w^{2}}{2 \sigma_{v}^{2}}\right) u(w),
$$

where $\sigma_{v}$ is the standard deviation of the signals $x_{n}$ before the absolute value operation, and $\sigma_{v}=E\left\{x_{n}^{2}\right\} . u(w)$ is a unit step function and $u(0)$ is set to $1 / 2$ in this paper.

Since the optical emitter is assumed to be ideal and the intensity of the output optical signal is proportional to the input electrical current, we can calculate the optical power of AVO-OFDM, which is given by

$$
P_{o, \text { avo }}=E\left\{x_{\text {avo }}(t)\right\}=\int_{0}^{+\infty} w f_{x_{\text {avo }}(t)}(w) d w=\frac{2 \sigma_{v}}{\sqrt{2 \pi}} .
$$

Meanwhile, the electrical power of AVO-OFDM is given by

$$
P_{e, \text { avo }}=E\left\{x_{\text {avo }}^{2}(t)\right\}=\int_{0}^{+\infty} w^{2} f_{x_{\text {avo }}(t)}(w) d w=\sigma_{v}^{2} .
$$

Similarly, we can obtain the PDF of ACO-OFDM timedomain signals $y_{\text {aco }}(t)$, which is given by [19]

$$
f_{y_{\text {aco }}(t)}(\nu)=\frac{1}{\sqrt{2 \pi} \sigma_{a}} \exp \left(\frac{-\nu^{2}}{2 \sigma_{a}^{2}}\right) u(\nu)+\frac{1}{2} \delta(\nu),
$$

where $\sigma_{a}$ is the standard deviation of the unclipped signal $y_{n}$, and $\sigma_{a}=\sqrt{E\left\{y_{n}^{2}\right\}}$ [19]. $\delta(\nu)$ is a unit impulse function. The optical and electrical power of ACO-OFDM time-domain signals are given by $P_{o \text {, aco }}=\sigma_{a} / \sqrt{2 \pi}$ and $P_{e, \text { aco }}=\sigma_{a}^{2} / 2$, respectively [19].

Theorem 1: When $N$ is large, $x_{\text {avo }}(t)$ and $y_{\text {aco }}(t)$ are approximately statistically independent.

Proof: See Appendix A.

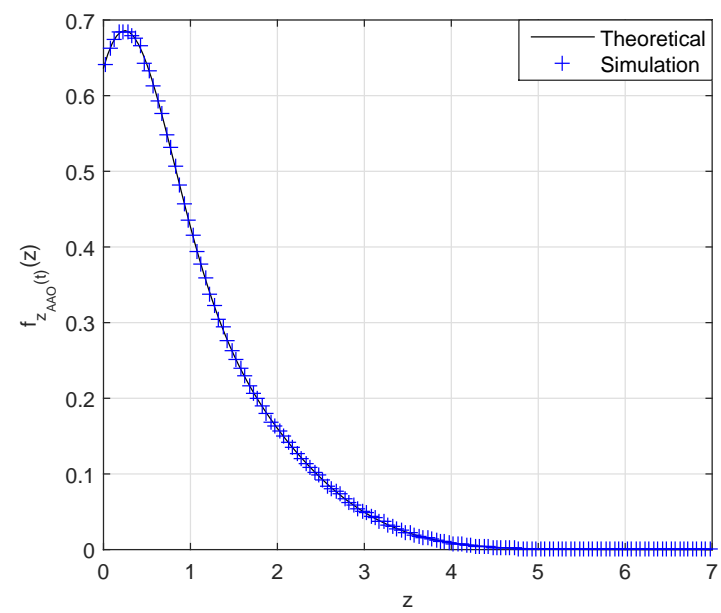

Fig. 3. The theoretical and simulated PDF of AAO-OFDM.

Therefore, the PDF of AAO-OFDM time-domain signal can be derived, which is given by

$$
\begin{aligned}
& f_{z_{\mathrm{aao}}(t)}(z)=\int_{-\infty}^{+\infty} f_{x_{\mathrm{avo}}(t)}(z-\lambda) f_{y_{\mathrm{aco}}(t)}(\lambda) d \lambda \\
= & \int_{0}^{z} \frac{\sqrt{2}}{\sqrt{\pi} \sigma_{v}} \exp \left(\frac{-(z-\lambda)^{2}}{2 \sigma_{v}^{2}}\right) \\
& \left(\frac{1}{\sqrt{2 \pi} \sigma_{a}} \exp \left(\frac{-\lambda^{2}}{2 \sigma_{a}^{2}}\right)+\frac{1}{2} \delta(\lambda)\right) d \lambda \\
= & \frac{1}{\sqrt{2 \pi} \sigma_{v}} \exp \left(\frac{-z^{2}}{2 \sigma_{v}^{2}}\right) u(z)+\frac{\sqrt{2}}{\sqrt{\pi} \sigma} \exp \left(\frac{-z^{2}}{2 \sigma^{2}}\right) \\
& \left\{Q\left(\frac{-z \sigma_{v}}{\sigma_{a} \sigma}\right)-Q\left(\frac{z \sigma_{a}}{\sigma_{v} \sigma}\right)\right\} u(z),
\end{aligned}
$$

where $Q(x)=\frac{1}{\sqrt{2 \pi}} \int_{x}^{+\infty} \exp \left(-\frac{x^{2}}{2}\right) d x$, and $\sigma$ is defined as $\sqrt{\sigma_{a}^{2}+\sigma_{v}^{2}}$.

The theoretical and simulated PDF of AAO-OFDM are shown in Fig. 3, where the average total optical power is set to unit and equal optical power allocation is used for AVO-OFDM and ACO-OFDM. In addition, the number of subcarriers $N$ is set to 1024 . It is shown that simulation results are aligned with the theoretical analysis according to (33) quite well.

The average optical power of AAO-OFDM time-domain signal is given by

$$
\begin{aligned}
P_{o, \text { aao }} & =E\left\{z_{\text {aao }}(t)\right\} \\
& =E\left\{x_{\text {avo }}(t)+y_{\text {aco }}(t)\right\} \\
& =\frac{2 \sigma_{v}+\sigma_{a}}{\sqrt{2 \pi}} .
\end{aligned}
$$

As discussed in Theorem 1, $x_{\mathrm{avo}}(t)$ and $y_{\mathrm{aco}}(t)$ are statistical independent when $N$ is large, we can estimate the average electrical power of AAO-OFDM time-domain signals, which 
is given by

$$
\begin{aligned}
P_{e, \text { aao }} & =E\left\{z_{\text {aao }}^{2}(t)\right\} \\
& =E\left\{\left(x_{\text {avo }}(t)+y_{\text {aco }}(t)\right)^{2}\right\} \\
& =E\left\{x_{\text {avo }}^{2}(t)\right\}+E\left\{y_{\text {aco }}^{2}(t)\right\}+2 E\left\{x_{\text {avo }}(t) y_{\text {aco }}(t)\right\} \\
& =\sigma_{v}^{2}+\frac{\sigma_{a}^{2}}{2}+\frac{2 \sigma_{v} \sigma_{a}}{\pi} .
\end{aligned}
$$

\section{B. The PAPR of AAO-OFDM Time-Domain Signals}

The implementation of optical OFDM is significantly influenced by the nonlinear transfer characteristics of the optical devices, such as LEDs, and it becomes more significant with larger PAPR. Thus the PAPR of AAO-OFDM time-domain signal $z_{\text {aao }}(t)$ is analyzed, which can be defined as [29]

$$
\operatorname{PAPR}_{\text {cont }}=\frac{\max _{t \in[0, T]} z_{\text {aao }}^{2}(t)}{E\left\{z_{\text {aao }}^{2}(t)\right\}},
$$

where $T$ is the symbol period. Although, the PAPR of the continuous time signal cannot be obtained precisely using the Nyquist rate sampling, an oversampling factor of four can provide sufficiently accurate results [29]. For simplicity, we investigate the PAPR of the discrete time signal $z_{\text {aao }, n}$ to estimate the performance of $\mathrm{PAPR}_{\text {cont }}$, as [27]

$$
\operatorname{PAPR}=\frac{\max _{0 \leq n \leq N-1} z_{\text {aao }, n}^{2}}{P_{e, \text { aаo }}} .
$$

Thus the cumulative distribution function (CDF) of PAPR is given by

$$
\operatorname{Pr}\{\text { PAPR } \leq \xi\}=\operatorname{Pr}\left\{\max _{0 \leq n \leq N-1} z_{\text {aao }, n}^{2} \leq \xi P_{e, \text { aao }}\right\},
$$

where $\operatorname{Pr}\{\Pi\}$ denotes the probability of the event $\Pi$.

Usually, we are interested in the probability of PAPR exceeding a certain level, thus the complementary cumulative distribution function (CCDF) of PAPR in AAO-OFDM is calculated, which is given by

$$
\operatorname{CCDF}(\xi)=1-\operatorname{Pr}\{\mathrm{PAPR} \leq \xi\},
$$

Theorem 2: When $N$ is large, the CCDF of the PAPR could be approximated as

$$
\operatorname{CCDF}(\xi)=1-\left\{1-2 Q\left(\frac{\sqrt{\xi P_{e, \text { aao }}}}{\sigma_{v}}\right)-2 A\right\}^{N / 2},
$$

where $A=\frac{2}{\sqrt{2 \pi} \sigma_{v}} \int_{0}^{\sqrt{\xi P_{e, \text { aa }}}} \exp \left(\frac{-\lambda^{2}}{2 \sigma_{v}^{2}}\right) Q\left(\frac{\sqrt{\xi P_{e, \text { aa }}}-\lambda}{\sigma_{a}}\right) d \lambda$.

Proof: See Appendix B.

The CCDFs of the PAPR in AAO-OFDM and other optical OFDM schemes are shown in Fig. 4, where $N$ is set to 1024. The CCDF of the PAPR in DCO-OFDM is calculated according to (11) in [27] and $\beta$ is set to $7.16 \mathrm{~dB}$ [19]. The CCDF of PAPR in ACO-OFDM is calculated according to (16) in [27]. For ADO-OFDM, the CCDF of its PAPR is given by simulation, where DCO-OFDM using 16QAM with $\beta=7.16 \mathrm{~dB}$ [19] and ACO-OFDM using 16QAM are employed. For ASCO-OFDM, ACO-OFDM using 16QAM

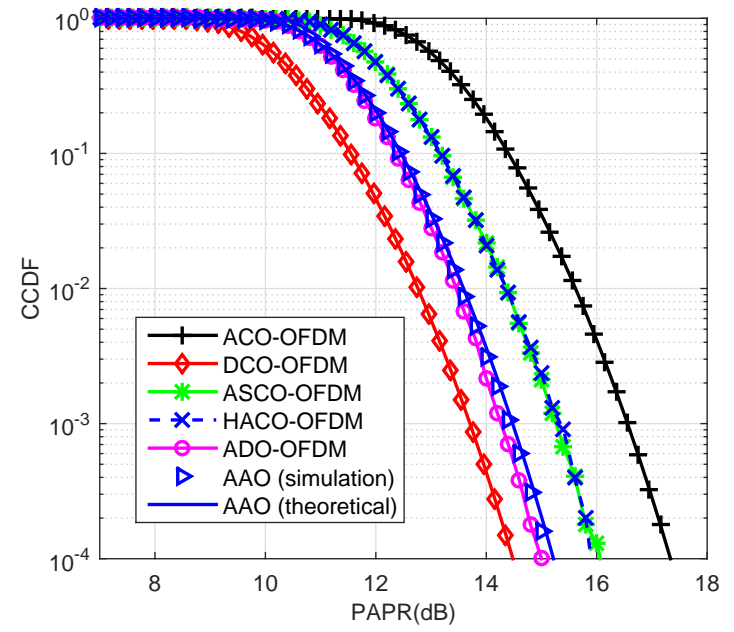

Fig. 4. CCDFs of PAPR in AAO-OFDM and its counterparts (the average total optical power of each optical OFDM scheme is set to unity and $N=$ 1024).

and SCO-OFDM using 16QAM are employed. For HACOOFDM, ACO-OFDM using 16QAM and PAM-DMT using $16 \mathrm{PAM}$ are utilized. And the CCDF of PAPR in AAO-OFDM is given via both simulation and theoretical analysis according to (39). In addition, the total average optical power of each OFDM scheme above is set to unit as in [19]. Compared to other optical OFDM schemes, ACO-OFDM has a higher PAPR due to the same peak power but lower average power caused by half of the time-domain signals been clipped at zeros [30]. For AAO-OFDM, the simulation results are aligned with the theoretical analysis as depicted in Fig. 4. When the CCDF approaches $10^{-4}$, the PAPR of AAO-OFDM is about $15.1 \mathrm{~dB}$, which is similar to ADO-OFDM and is $2.1 \mathrm{~dB}, 0.9 \mathrm{~dB}$ and $0.7 \mathrm{~dB}$ lower compared to ACO-OFDM, ASCO-OFDM and HACO-OFDM, respectively. This indicates that AAO-OFDM is less sensitive to the nonlinearity of the OWC devices. Although the PAPR of DCO-OFDM is lower compared to AAO-OFDM, DCO-OFDM is less attractive, since the DC bias is required.

\section{Spectral Efficiency Analysis}

For AAO-OFDM, there are $\left(\frac{N}{4}-1\right)$ effective complex symbols before Hermitian symmetry for AVO-OFDM, and $\left(\frac{N}{4}-N_{\text {sign }}\right)$ useful complex symbols with $N_{\text {sign }}$ sign symbols for ACO-OFDM. The data structures of $\mathbf{X}$ and $\mathbf{Y}$ with $N=16$ are shown in Fig. 5 for example.

Thus the spectral efficiency of AAO-OFDM is denoted by $\Upsilon_{\mathrm{AAO}}$, given by

$$
\begin{aligned}
\Upsilon_{\mathrm{AAO}}= & \frac{\left(\frac{N}{4}-1\right) \log _{2} M_{1}+\left(\frac{N}{4}-\left\lceil\frac{N / 2}{\log _{2} M_{\text {sign }}}\right]\right) \log _{2} M_{2}}{N} \\
= & \frac{1}{4}\left(\log _{2} M_{1}+\log _{2} M_{2}\right)-\left\lceil\frac{N / 2}{\log _{2} M_{\text {sign }}}\right] \frac{\log _{2} M_{2}}{N} \\
& -\frac{1}{N} \log _{2} M_{1} \mathrm{bit} / \mathrm{s} / \mathrm{Hz} .
\end{aligned}
$$

When $N$ is large and the same constellation size is utilized 
TABLE I

THE SPECTRAL EFFICIENCY COMPARISON BETWEEN AAO-OFDM AND ITS COUNTERPARTS

\begin{tabular}{cccccccccc}
\hline \hline Constellation size groups & $\Upsilon_{\mathrm{AAO}}^{\mathrm{appr}}$ & $\Upsilon_{\mathrm{DCO}}^{\mathrm{appr}}$ & $\Upsilon_{\mathrm{ADO}}^{\mathrm{appr}}$ & $\Upsilon_{\mathrm{ASCO}}^{\mathrm{appr}}$ & $\Upsilon_{\mathrm{ACO}}$ & $\Upsilon_{\mathrm{AAO}}$ & $\Upsilon_{\mathrm{DCO}}$ & $\Upsilon_{\mathrm{ADO}}$ & $\Upsilon_{\mathrm{ASCO}}$ \\
\hline A (odd 4QAM \& even 4QAM) & 0.500 & 1.000 & 1.000 & 0.750 & 0.500 & 0.498 & 0.998 & 0.998 & 0.748 \\
B (odd 8QAM \& even 8QAM) & 1.000 & 1.500 & 1.500 & 1.125 & 0.750 & 0.996 & 1.497 & 1.497 & 1.122 \\
C (odd 16QAM \& even 16QAM) & 1.500 & 2.000 & 2.000 & 1.500 & 1.000 & 1.496 & 1.996 & 1.996 & 1.496 \\
D (odd 32QAM \& even 32QAM) & 2.000 & 2.500 & 2.500 & 1.875 & 1.250 & 1.992 & 2.495 & 2.495 & 1.870 \\
E (odd 64QAM \& even 64QAM) & 2.500 & 3.000 & 3.000 & 2.350 & 1.500 & 2.490 & 2.994 & 2.994 & 2.244 \\
F (odd 128QAM \& even 128QAM) & 3.000 & 3.500 & 3.500 & 2.625 & 1.750 & 2.987 & 3.493 & 3.493 & 2.618 \\
G (odd 256QAM \& even 256QAM) & 3.500 & 4.000 & 4.000 & 3.000 & 2.000 & 3.492 & 3.992 & 3.992 & 2.992 \\
H (odd 512QAM \& even 512QAM) & 4.000 & 4.500 & 4.500 & 3.375 & 2.250 & 3.990 & 4.491 & 4.491 & 3.366 \\
I (odd 1024QAM \& even 1024QAM) & 4.500 & 5.000 & 5.000 & 3.750 & 2.500 & 4.482 & 4.990 & 4.990 & 3.740 \\
\hline \hline
\end{tabular}

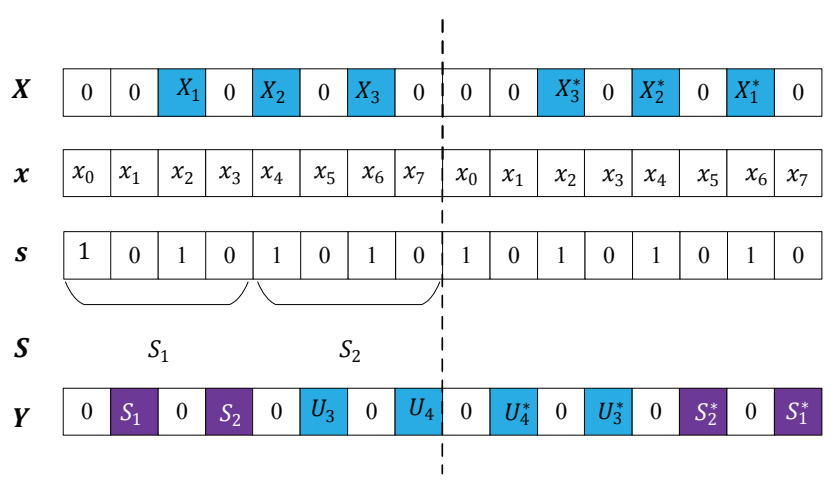

Fig. 5. Data structures of important data vectors $\mathbf{X}$ and $\mathbf{Y}$ with $N=16$ for example.

on the odd subcarriers, i.e. $M_{\text {sign }}=M_{2}$, an approximation of $\Upsilon_{\mathrm{AAO}}$ is given by

$$
\Upsilon_{\mathrm{AAO}}^{\mathrm{appr}} \approx \frac{1}{4}\left(\log _{2} M_{1}+\log _{2} M_{2}\right)-\frac{1}{2} \mathrm{bit} / \mathrm{s} / \mathrm{Hz} .
$$

Similarly, we could calculate the spectral efficiency of other optical OFDM schemes. When constellation sizes $M_{1}$ and $M_{2}$ are utilized in the even and odd subcarriers, the spectral efficiency of ADO-OFDM, DCO-OFDM, ASCO-OFDM and ACO-OFDM are given by

$$
\begin{aligned}
& \Upsilon_{\mathrm{ADO}}=\frac{1}{4}\left(\log _{2} M_{1}+\log _{2} M_{2}\right)-\frac{1}{N} \log _{2} M_{1} \mathrm{bit} / \mathrm{s} / \mathrm{Hz}, \\
& \Upsilon_{\mathrm{DCO}}=\frac{1}{4}\left(\log _{2} M_{1}+\log _{2} M_{2}\right)-\frac{1}{N} \log _{2} M_{1} \mathrm{bit} / \mathrm{s} / \mathrm{Hz},
\end{aligned}
$$

$$
\Upsilon_{\mathrm{ASCO}}=\frac{1}{4}\left(\frac{1}{2} \log _{2} M_{1}+\log _{2} M_{2}\right)-\frac{1}{N} \log _{2} M_{1} \mathrm{bit} / \mathrm{s} / \mathrm{Hz}
$$

and

$$
\Upsilon_{\mathrm{ACO}}=\frac{1}{4}\left(\log _{2} M_{2}\right) \mathrm{bit} / \mathrm{s} / \mathrm{Hz}
$$

Note that, when $N$ is large, the last term in (43), (44) and (45) are so small that can be negligible, which lead to the corresponding approximations as

$$
\Upsilon_{\mathrm{ADO}}^{\mathrm{appr}} \approx \frac{1}{4}\left(\log _{2} M_{1}+\log _{2} M_{2}\right) \mathrm{bit} / \mathrm{s} / \mathrm{Hz}
$$

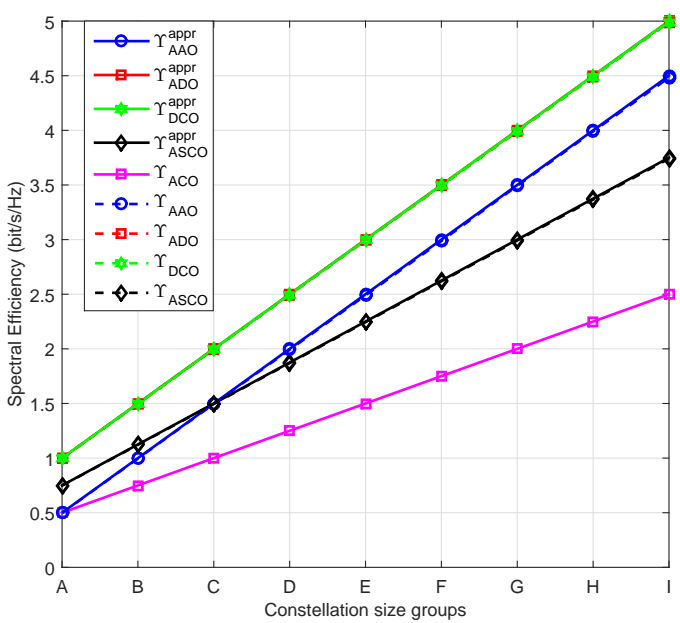

Fig. 6. The spectral efficiency comparison between AAO-OFDM and its counterparts ('A' $\sim$ 'I' denote the constellation groups defined in Table I).

$$
\Upsilon_{\mathrm{DCO}}^{\mathrm{appr}} \approx \frac{1}{4}\left(\log _{2} M_{1}+\log _{2} M_{2}\right) \mathrm{bit} / \mathrm{s} / \mathrm{Hz}
$$

and

$$
\Upsilon_{\mathrm{ASCO}}^{\mathrm{appr}} \approx \frac{1}{4}\left(\frac{1}{2} \log _{2} M_{1}+\log _{2} M_{2}\right) \mathrm{bit} / \mathrm{s} / \mathrm{Hz}
$$

The spectral efficiency comparison between AAO-OFDM and its counterparts is shown in Table I and Fig. 6, where $N=$ 1024 is assumed in (41), (43), (44) and (45). In AAO-OFDM, $M_{\text {sign }}$ is assumed to equal to $M_{2}$ for simplicity. As shown in Fig. 6, for each optical OFDM scheme, the difference between the spectral efficiency and its approximation value is so small that can be negligible. In addition, it indicates that $\Upsilon_{\mathrm{AAO}}$ is about 0.5 bits $/ \mathrm{s} / \mathrm{Hz}$ less compared to $\Upsilon_{\mathrm{ADO}}$ and $\Upsilon_{\mathrm{DCO}}$ with the same constellation sizes. However, no DC bias is required for AAO-OFDM, which is energy efficient. In addition, $\Upsilon_{\text {AAO }}$ is $\left(\frac{1}{8} \log _{2} M_{2}-\frac{1}{2}\right)$ bits/s/Hz larger compared to $\Upsilon_{\mathrm{ASCO}}$, and $\left(\frac{1}{4} \log _{2} M_{2}-\frac{1}{2}\right)$ bits/s/Hz larger compared to $\Upsilon_{\text {ACO }}$ with the same constellation sizes.

\section{Optical Power Allocation Discussion}

The ACO-OFDM symbols are only distorted by the noise falling on the odd subcarriers, hence they are directly detected 
TABLE II

The Computational Complexity ANALYsis of AAO-OFDM AND ITS COUNTERPARTS.

\begin{tabular}{ccc}
\hline \hline OFDM schemes & Transmitter & Receiver \\
\hline DCO-OFDM & $\mathcal{O}\left(N \log _{2} N\right)$ & $\mathcal{O}\left(N \log _{2} N\right)$ \\
ACO-OFDM & $\mathcal{O}\left(N \log _{2} N\right)$ & $\mathcal{O}\left(N \log _{2} N\right)$ \\
ADO-OFDM & $2 \mathcal{O}\left(N \log _{2} N\right)$ & $4 \mathcal{O}\left(N \log _{2} N\right)$ \\
HACO-OFDM & $2 \mathcal{O}\left(N \log _{2} N\right)$ & $3 \mathcal{O}\left(N \log _{2} N\right)$ \\
ASCO-OFDM & $2 \mathcal{O}\left(N \log _{2} N\right)$ & $3 \mathcal{O}\left(N \log _{2} N\right)$ \\
AAO-OFDM & $2 \mathcal{O}\left(N \log _{2} N\right)$ & $4 \mathcal{O}\left(N \log _{2} N\right)$ \\
\hline \hline
\end{tabular}

at the receiver. While the AVO-OFDM symbols are recovered after the local estimation of ACO-OFDM signals are removed, using the signs estimators, $\left\{\widehat{S}_{k}\right\}$, which are estimated from the ACO-OFDM part. Thus, the performance of AVO-OFDM is degraded by the noise, the estimation error of the ACO-OFDM negative clipping distortion and the estimation error of $\left\{\widehat{S}_{k}\right\}$. Fortunately, when the SNR increases, the estimation of the clipping distortion and $\left\{\widehat{S}_{k}\right\}$ become more accurate, which cause smaller performance degradation to the AVO-OFDM part. In addition, numerical results under equal optical power allocation are desirable, and the performance of AVO-OFDM part is similar to ACO-OFDM in the high SNR regime, which is preferable in a practical system. Therefore, the equal optical power allocation between AVO-OFDM and ACO-OFDM is considered in this paper.

\section{E. Computational Complexity Analysis}

At the transmitter of AAO-OFDM, two $N$-point IFFT blocks are utilized as shown in Fig. 1, thus its computational complexity can be written as $2 \mathcal{O}\left(N \log _{2} N\right)$ [31]. While at the receiver, two $N$-point FFT blocks with two $N$-point IFFT blocks are required as shown in Fig. 2, and the multipliers can be replaced by shifting $\pi$ phases, since the sign is either ' 1 ' or ' -1 '. Hence the computational complexity of the receiver can be written as $4 \mathcal{O}\left(N \log _{2} N\right)$. For ADO-OFDM, two $N$-point IFFT blocks are employed in the transmitter and two $N$-point FFT blocks with two $N$-point IFFT blocks at the receiver [18], [19], thus the computational complexity can be estimated by $2 \mathcal{O}\left(N \log _{2} N\right)$ for its transmitter and $4 \mathcal{O}\left(N \log _{2} N\right)$ for its receiver. Similarly, for DCO-OFDM and ACO-OFDM, one $N$-point IFFT block and one $N$-point FFT block are employed at the transceiver for both of the schemes. Hence the computational complexity can be estimated by $\mathcal{O}\left(N \log _{2} N\right)$ for both the transmitter and the receiver. For HACO-OFDM and ASCO-OFDM, two $N$-point IFFT blocks are utilized for the transmitter and two $N$-point FFT blocks plus one $N$-point IFFT block are used at the receiver, whose computational complexity can be written as $2 \mathcal{O}\left(N \log _{2} N\right)$ for the transmitter and $3 \mathcal{O}\left(N \log _{2} N\right)$ for the receiver. The computational complexity analysis of the six optical OFDM schemes are presented in Table II. Evidently, the computational complexity of AAO-OFDM is similar to that of ADO-OFDM, which is slightly higher than its counterparts but acceptable considering its benefits such as DC bias elimination, higher energy and spectral efficiency and lower PAPR.

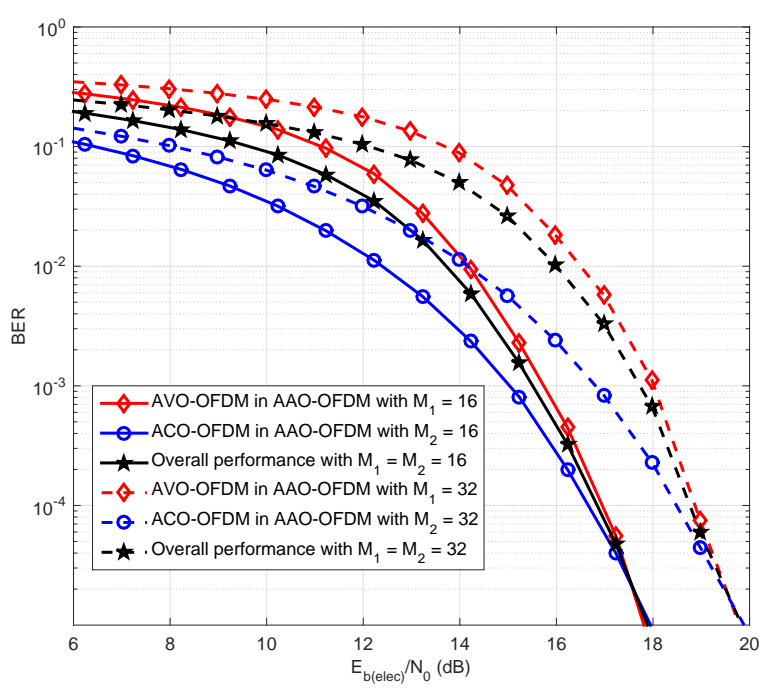

Fig. 7. The BER performance of AAO-OFDM with different spectral efficiencies.

\section{NumERicAl RESUlts}

The BER performance of AAO-OFDM is analyzed and compared to other optical OFDM schemes in terms of electrical bit energy to noise power ratio $E_{b} / N_{0}$. The average total optical power of each OFDM scheme is normalized to unity [19]. Meanwhile, equal optical power allocation between AVO-OFDM and ACO-OFDM is employed according the analysis in Section IV-D. For simplicity, the same constellation size is used for the odd subcarriers, i.e. $M_{\text {sign }}=M_{2}$. In this paper, a flat AWGN channel with perfect equalization and ideal time synchronization is assumed [18], [19]. In addition, the FFT/IFFT size is set to be 1024 , i.e. $N=1024$.

The BER performance of AAO-OFDM with different spectral efficiencies is shown in Fig. 7, where $M_{1}=M_{2}=16$ for spectral efficiency $\Upsilon=1.5 \mathrm{bit} / \mathrm{s} / \mathrm{Hz}$ and $M_{1}=M_{2}=32$ for $\Upsilon=2.0 \mathrm{bit} / \mathrm{s} / \mathrm{Hz}$ are utilized, respectively. Based on the analysis in Section IV-D, equal optical power allocation between AVO-OFDM and ACO-OFDM is utilized. Firstly, it can be seen that the symbols modulated in AAO-OFDM could be detected successfully with the receiver. When $E_{b} / N_{0}$ is small, the BER of AVO-OFDM is higher compared to that of ACO-OFDM, since the symbols in AVO-OFDM are not only distorted by noise but also by the estimation errors of ACOOFDM clipping distortion and sign symbols $\left\{\widehat{S}_{k}\right\}$. However, when $E_{b} / N_{0}$ increases, the estimation accuracy of ACOOFDM symbols improves with the reduction of the BER, and the signs of AVO-OFDM could be estimated more accurately, resulting in improvement of AVO-OFDM BER performance. In addition, when $E_{b} / N_{0}$ is high, the performances of AVOOFDM and ACO-OFDM are similar at a BER of $10^{-5}$. The results are desirable since the information transmitted by AVO-OFDM and ACO-OFDM is preferred to have similar performance in practical communication systems.

Although the spectral efficiencies of different OFDM schemes are different with the same constellation sizes, we can utilize different constellation combinations to achieve the 


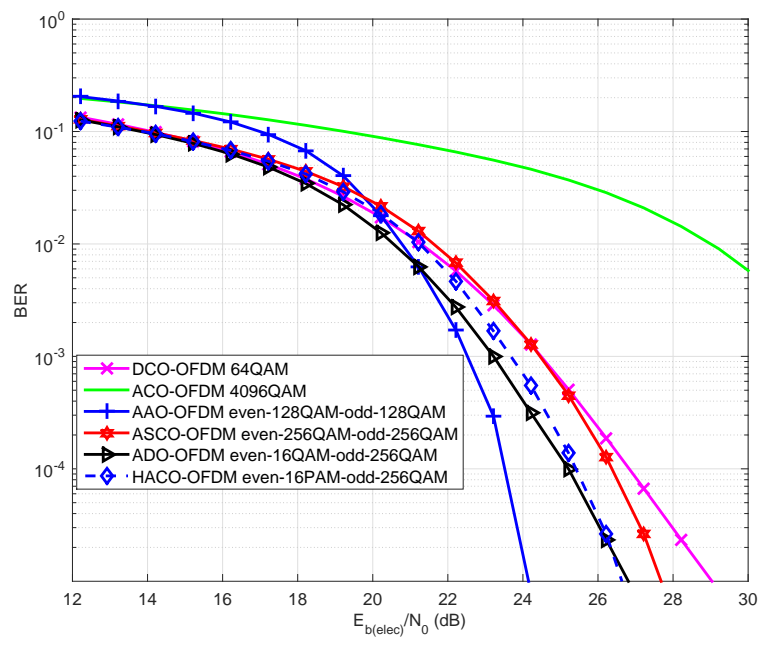

Fig. 8. BER performance comparison between AAO-OFDM and its counterparts with the same spectral efficiency (the average total optical power of each OFDM scheme is normalized to unity).

same spectral efficiency as shown in Table I and Fig. 6. Without loss of generality, the BER performance of AAOOFDM and its counterparts with $\Upsilon=3 \mathrm{bit} / \mathrm{s} / \mathrm{Hz}$ are compared in Fig. 8. For DCO-OFDM with 64QAM, the DC bias-index $\beta$ is set to $8.99 \mathrm{~dB}$ [19]. For ACO-OFDM, 4096QAM is employed. For AAO-OFDM, 128QAM is used in both the even and odd subcarriers, and equal optical power allocation is imposed. To enhance readability, $\alpha$ is defined to denote the optical power allocation factor for the subsystem used in odd subcarriers in each scheme, and for AAO-OFDM, $\alpha=0.5$. For ASCO-OFDM, 256QAM is utilized in both the even and odd subcarriers with the power allocation factor $\alpha=0.5$. For ADO-OFDM, four constellation combinations with different DC biases and different optimal power allocation factors are used to find its best performance. Specifically, even-64QAM-odd-64QAM, even-32QAM-odd-128QAM, even-16QAM-odd-256QAM, and even-8QAM-odd-512QAM are employed with $\beta$ equal to $8.99 \mathrm{~dB}$ [19], $8.00 \mathrm{~dB}, 7.16 \mathrm{~dB}$ and $6.80 \mathrm{~dB}$, respectively. In addition, $\alpha$ are correspondingly set to $0.18,0.33,0.53$ and 0.71 , calculated according to (33) in [32]. Among these four constellation combinations, the simulation results show that ADO-OFDM using even-16QAModd-256QAM with $\beta=7.16 \mathrm{~dB}$ and $\alpha=0.53$ has the best performance at a BER of $10^{-5}$. Similarly, for HACO-OFDM, four constellation combinations with different optimal power allocation factors are also used to find the best performance. More specifically, even-64PAM-odd-64QAM, even-32PAModd-128QAM, even-16PAM-odd-256QAM, and even-8PAModd-512QAM are employed, and $\alpha$ equaling $0.16,0.34,0.59$ and 0.80 , respectively, calculated according to (22) in [16]. The simulation results show that HACO-OFDM employing even-16PAM-odd-256QAM with $\beta=6.46 \mathrm{~dB}$ and $\alpha=0.59$ has the best BER performance when BER approaches to $10^{-5}$ among these four constellation combinations.

Figure 8 indicates that AAO-OFDM with equal optical power allocation achieves the best BER performance among these optical OFDM schemes when the BER is less than $10^{-5}$. More specifically, AAO-OFDM achieves $4.9 \mathrm{~dB}$ and $2.7 \mathrm{~dB}$ performance gains than DCO-OFDM with $\beta=8.99$ $\mathrm{dB}$ and ADO-OFDM using even-16QAM-odd-256QAM with $\beta=7.16 \mathrm{~dB}$ and $\alpha=0.53$ at a BER of $10^{-5}$, respectively. Since no DC bias is required for AAO-OFDM, and its spectral efficiency is only $0.5 \mathrm{bit} / \mathrm{s} / \mathrm{Hz}$ less than DCO-OFDM and ADO-OFDM according to Fig. 6, so that its constellation size is only slightly larger to achieve the same data rate. In addition, AAO-OFDM uses both the real and imaginary parts of even and odd subcarriers and is more spectrally efficient, its constellation size is much smaller than ACOOFDM, ASCO-OFDM and HACO-OFDM given the same spectral efficiency. Therefore, AAO-OFDM achieves $11.3 \mathrm{~dB}$, $3.5 \mathrm{~dB}$ and $2.5 \mathrm{~dB}$ performance gains than ACO-OFDM, ASCO-OFDM, and HACO-OFDM using even-16PAM-odd256QAM with $\alpha=0.59$ at a BER of $10^{-5}$, respectively.

\section{CONCLUSIONS}

In this paper, we propose a novel energy- and spectrumefficient scheme called AAO-OFDM for IM/DD based OWC systems, where AVO-OFDM signals on the even subcarriers and ACO-OFDM signals on the odd subcarriers are combined for simultaneous transmission. AAO-OFDM could employ all the subcarriers like DCO-OFDM while requiring no DC biases like ACO-OFDM at the same time. Simulation results validate the theoretical analysis and indicate that AAO-OFDM has better energy efficiency than DCO-OFDM and ADO-OFDM, and better spectrum efficiency than ACO-OFDM, ASCOOFDM and HACO-OFDM. Meanwhile, it has lower PAPR and performs better compared to those conventional methodologies given the same spectral efficiency in the high SNR region.

In this paper, we only concentrate on the transceiver design of the proposed AAO-OFDM and analyze its corresponding performance. In the future, we plan to find the optimal optical power allocation between AVO-OFDM and ACO-OFDM signals, investigate the effects of chromatic dispersion and the nonlinearity of the LEDs in order to futher improve the performance in practical IM/DD systems.

\section{APPENDIX A \\ PROOF OF THEOREM 1}

Firstly, we assume a continuous random variable $X$ with zero mean and the symmetric $\operatorname{PDF} f_{X}(x)$, i.e. $f_{X}(x)=f_{X}(-x)$.

We assume $Y=|X|$, and its PDF can be derived easily as $f_{Y}(y)=2 f_{X}(y) u(y)$, where $u(y)$ is a unit step function with $u(0)=\frac{1}{2}$. Thus we can calculate the probability of $\operatorname{Pr}\{Y \leq$ $y\}$, which is given by

$$
\begin{aligned}
\operatorname{Pr}\{Y \leq y\} & =\operatorname{Pr}\{|X| \leq y\} \\
& =\operatorname{Pr}\{-y \leq X \leq y\} \\
& =2 \operatorname{Pr}\{0 \leq X \leq y\} .
\end{aligned}
$$

We also assume a variable $S$ denoting the sign of $X$, which is given by

$$
S= \begin{cases}1, & X \geq 0 \\ 0, & X<0 .\end{cases}
$$

It is easy to derive that $\operatorname{Pr}\{S=1\}=\operatorname{Pr}\{S=0\}=0.5$. 
Based on the former assumptions, we have

$$
\begin{aligned}
\operatorname{Pr}\{S=1, Y \leq y\} & =\operatorname{Pr}\{x \geq 0,|x| \leq y\} \\
& =\operatorname{Pr}\{0 \leq x \leq y\} \\
& =\operatorname{Pr}\{S=1\} \operatorname{Pr}\{Y \leq y\},
\end{aligned}
$$

and

$$
\begin{aligned}
\operatorname{Pr}\{S=0, Y \leq y\} & =\operatorname{Pr}\{x<0,|x| \leq y\} \\
& =\operatorname{Pr}\{-y \leq x<0\} \\
& =\operatorname{Pr}\{S=0\} \operatorname{Pr}\{Y \leq y\} .
\end{aligned}
$$

Thus, $Y$ and $S$ are statistically independent.

As discussed in Section IV-A, the bipolar time-domain signals $x(t)$ follows a Gaussian distribution with zero mean, which has a symmetric PDF [17], [27], [28]. This holds for large subcarrier numbers especially when $N \geq 128$ [17], [27]. Thus, the absolute value of $x(t)$ and its sign are approximately statistically independent. Therefore, although $y_{\text {aco }}(t)$ consists of the signs related to $x(t), x_{\text {avo }}(t)$ and $y_{\text {aco }}(t)$ are approximately statistically independent when $N$ is large.

\section{APPENDIX B \\ PROOF OF THEOREM 2}

Since $\left\{x_{n}\right\}$ have the symmetry property, i.e. $x_{n}=$ $x_{n+N / 2}, 0 \leq n \leq N / 2-1, x_{n}$ and $x_{n+N / 2}$ are pairwise correlated. In addition, using the central limit theorem, the bipolar time-domain signal $x_{n}$ follows a Gaussian distribution with zero mean. Besides, the correlation among $\left\{x_{n}, n=\right.$ $0,1, \ldots, N / 2-1\}$ tends to zero for large $N$ [33]. Thus, $\left\{x_{n}, n=0,1, \ldots, N / 2-1\right\}$ can be treated as independent for large $N$. Based on the analysis above, we can conclude that $\left\{x_{\mathrm{avo}, n}, n=0,1, \ldots, N / 2-1\right\}$ are independent for large $N$, where $x_{\mathrm{avo}, n}$ is assumed to be the sample of signal $x_{\mathrm{avo}}(t)$, and $x_{\mathrm{avo}, n}$ and $x_{\mathrm{avo}, n+N / 2}$ are pairwise correlated. So do $\left\{y_{\text {aco }, n}, n=0,1, \ldots, N / 2-1\right\}$.

We assume that $z_{\text {aao }, n}$ is the sample of $z_{\text {aao }}(t)$, and $z_{\text {aao }, n}=$ $x_{\mathrm{avo}, n}+y_{\mathrm{aco}, n}$. According to Theorem $1, x_{\mathrm{avo}, n}$ and $y_{\mathrm{aco}, n}$ are statistically independent when $N$ is large. Thus, based on the analysis above, we can conclude that $z_{\mathrm{aao}, n}$ and $z_{\mathrm{aao}, n+N / 2}$ are also pairwise correlated and $\left\{z_{\text {aao }, n}, n=0,1, \ldots, N / 2-1\right\}$ are independent when $N$ is large.

Hence, the CDF of the AAO-OFDM PAPR is given by

$$
\begin{aligned}
& \operatorname{Pr}(\operatorname{PAPR} \leq \xi)=\operatorname{Pr}\left\{\max _{0 \leq n \leq N-1} z_{\text {aao }, n}^{2} \leq \xi P_{e, \text { aao }}\right\} \\
= & \operatorname{Pr}\left\{z_{\text {aao }, n} \leq \sqrt{\xi P_{e, \text { aao }}}, n=0,1, \cdots, N-1\right\} \\
= & \operatorname{Pr}\left\{z_{\text {aao }, n} \leq \sqrt{\xi P_{e, \text { aao }}}, z_{\text {aao }, n+\frac{N}{2}} \leq \sqrt{\xi P_{e, \text { aao }}},\right. \\
& n=0,1, \cdots, N / 2-1\} \\
& N / 2-1 \\
= & \prod_{n=0} \operatorname{Pr}\left\{z_{\text {aao }, n} \leq \sqrt{\xi P_{e, \text { aao }}}, z_{\text {aao }, n+\frac{N}{2}} \leq \sqrt{\xi \operatorname{Pr}_{e, \text { aao }}}\right\} .
\end{aligned}
$$

It follows that

$$
\begin{aligned}
& \operatorname{Pr}\left\{z_{\mathrm{aao}, n} \leq \sqrt{\xi P_{e, \mathrm{aao}}}, z_{\mathrm{aao}, n+\frac{N}{2}} \leq \sqrt{\xi P_{e, \mathrm{aao}}}\right\} \\
= & \operatorname{Pr}\left\{x_{\mathrm{avo}, n}+y_{\mathrm{aco}, n} \leq \sqrt{\xi P_{e, \mathrm{aao}}}, y_{n}>0\right\} \\
& +\operatorname{Pr}\left\{x_{\mathrm{avo}, n+\frac{N}{2}}+y_{\mathrm{aco}, n+\frac{N}{2}} \leq \sqrt{\xi P_{e, \mathrm{aao}}}, y_{n} \leq 0\right\} \\
\stackrel{(a)}{=} & 2 \operatorname{Pr}\left\{x_{\mathrm{avo}, n}+y_{\mathrm{aco}, n} \leq \sqrt{\xi P_{e, \mathrm{aao}}}, y_{n}>0\right\},
\end{aligned}
$$

where $(a)$ is because $y_{n}>0$ and $y_{n} \leq 0$ have the same distribution, i.e. $\operatorname{Pr}\left\{y_{n}>0\right\}=\operatorname{Pr}\left\{y_{n} \leq 0\right\}=0.5$.

According to the analysis in Section IV-A, we have

$$
\begin{aligned}
& \operatorname{Pr}\left\{x_{\mathrm{avo}, n}+y_{\mathrm{aco}, n} \leq \sqrt{\xi P_{e, \mathrm{aao}}}, y_{n}>0\right\} \\
= & \int_{0}^{\tau} d z \int_{0}^{z} \frac{\sqrt{2}}{\sqrt{\pi} \sigma_{v}} \exp \left(\frac{-\lambda^{2}}{2 \sigma_{v}^{2}}\right) \frac{1}{\sqrt{2 \pi} \sigma_{a}} \exp \left(\frac{-(z-\lambda)^{2}}{2 \sigma_{a}^{2}}\right) d \lambda \\
= & 0.5-Q\left(\frac{\tau}{\sigma_{v}}\right)-\frac{2}{\sqrt{2 \pi} \sigma_{v}} \int_{0}^{\tau} \exp \left(\frac{-\lambda^{2}}{2 \sigma_{v}^{2}}\right) Q\left(\frac{\tau-\lambda}{\sigma_{a}}\right) d \lambda,
\end{aligned}
$$

where $\tau=\sqrt{\xi P_{e, \text { aao }}}$, and $P_{e, \text { aao }}$ is given by (35).

Hence, the CDF of the PAPR for AAO-OFDM is derived as

$$
\begin{aligned}
& \operatorname{Pr}(\operatorname{PAPR} \leq \xi) \\
= & \left\{2 \operatorname{Pr}\left\{x_{\mathrm{avo}, n}+y_{\mathrm{aco}, n} \leq \sqrt{\xi P_{e, \mathrm{aao}}}, y_{n}>0\right\}\right\}^{N / 2} \\
= & \left\{1-2 Q\left(\frac{\sqrt{\xi P_{e, \mathrm{aao}}}}{\sigma_{v}}\right)-2 A\right\}^{N / 2},
\end{aligned}
$$

where $A=\frac{2}{\sqrt{2 \pi} \sigma_{v}} \int_{0}^{\sqrt{\xi P_{e, \text { aао }}}} \exp \left(\frac{-\lambda^{2}}{2 \sigma_{v}^{2}}\right) Q\left(\frac{\sqrt{\xi P_{e, \text { aаo }}}-\lambda}{\sigma_{a}}\right) d \lambda$.

Therefore, the CCDF of the PAPR for AAO-OFDM is given by

$$
\begin{aligned}
\operatorname{CCDF}(\xi) & =1-\operatorname{Pr}(\operatorname{PAPR} \leq \xi) \\
& =1-\left\{1-2 Q\left(\frac{\sqrt{\xi P_{e, \text { aao }}}}{\sigma_{v}}\right)-2 A\right\}^{N / 2},
\end{aligned}
$$

which leads to Theorem 2.

\section{REFERENCES}

[1] P. H. Pathak, X. Feng, P. Hu, and P. Mohapatra, "Visible light communication, networking, and sensing: a survey, potential and challenges", IEEE Commun. Surveys Tuts., vol. 17, no. 4, pp. 2047-2077, Sep. 2015.

[2] L. Hanzo, H. Haas, S. Imre, D. C. O’Brien, M. Rupp, and L. Gyongyosi, "Wireless myths, realities, and futures: from $3 \mathrm{G} / 4 \mathrm{G}$ to optical and quantum wireless," Proc. IEEE, vol. 100, no. 13, pp. 1853-1888, May 2012.

[3] C. Gong, S. Li, Q. Gao, and Z. Xu, "Power and rate optimization for visible light communication system with lighting constraints," IEEE Trans. Signal Process., vol. 63, no. 16, pp. 4245-4256, Aug. 2015.

[4] J. Zhou, Y. Yan, Z. Cai, Y. Qiao, and Y. Ji, "A cost-effective and efficient scheme for optical OFDM in short-range IM/DD systems," IEEE Photon. Technol. Lett., vol. 26, no. 13, pp. 1372-1374, Jul. 2014.

[5] G. Pedersen, "Amplitude modulated RF fields stemming from a GSM/DCS-1800 phone," Wireless Netw., vol. 3, no. 6, pp. 489-498, Nov. 1997.

[6] A. Chubukjian, J. Benger, R. Otnes, and B. Kasper, "Potential effects of broadband wireline telecommunications on the HF spectrum," IEEE Commun. Mag., vol. 46, no. 11, pp. 49-54, Nov. 2008.

[7] X. Liu, C. Gong, S. Li, and Z. Xu, "Signal characterization and receiver design for visible light communication under weak illuminance," IEEE Commun. Lett., vol. 20, no. 7, pp. 1349-1352, Jul. 2016. 
[8] J. Zhou, Y. Qiao, T. Zhang, E. Sun, M. Guo, Z. Zhang, X. Tang, and F. $\mathrm{Xu}$, "FOFDM based on discrete cosine transform for intensity-modulated and direct-detected systems." J. Lightw. Technol., vol. 34, no. 16, pp. 3717-3725, Aug. 2016.

[9] R. Mesleh, H. Elgala, and H. Haas, "LED nonlinearity mitigation techniques in optical wireless OFDM communication systems," J. Opt. Commun. Netw., vol. 4, no. 11, pp. 865-875, Nov. 2012.

[10] L. Peng, S. Haese and M. Helard, "Frequency domain LED compensation for nonlinearity mitigation in DMT systems," IEEE Photon. Technol. Lett., vol. 25, no. 20, pp. 2022-2025, Oct. 15, 2013.

[11] J. Tan, Z. Wang, Q. Wang, and L. Dai, "BICM-ID scheme for clipped DCO-OFDM in visible light communications," Opt. Express, vol. 24, no. 5, pp. 4573-4581, Feb. 2016

[12] R. Zhang and L. Hanzo, "Multi-layer modulation for intensity-modulated direct-detection optical OFDM," J. Opt. Commun. Netw., vol. 5, no. 12, pp. 1402-1412, Dec. 2013.

[13] A. M. Khalid, G. Cossu, R. Corsini, P. Choudhury, and E. Ciaramella, " $1-\mathrm{Gb} / \mathrm{s}$ transmission over a phosphorescent white LED by using rateadaptive discrete multitone modulation," IEEE Photon. J., vol. 4, no. 5 , pp. 1465-1473, Jul. 2012.

[14] B. Ranjha and M. Kavehrad, "Hybrid asymmetrically clipped OFDMbased IM/DD optical wireless system," J. Opt. Commun. Netw., vol. 6, no. 4, pp. 387-396, Apr. 2014.

[15] J. Armstrong and A. J. Lowery, "Power efficient optical OFDM," Electron. Lett., vol. 42, no. 6, pp. 370-372, Mar. 2006.

[16] Q. Wang, Z. Wang and L. Dai, "Iterative receiver for hybrid asymmetrically clipped optical OFDM," J. Lightw. Technol., vol. 32, no. 22, pp. 4471-4477, Nov. 2014.

[17] J. Armstrong, "OFDM for optical communications," J. Lightw. Technol., vol. 27, no. 3, pp. 189-204, Feb. 2009.

[18] S. D. Dissanayake, K. Panta and J. Armstrong, "A novel technique to simultaneously transmit ACO-OFDM and DCO-OFDM in IM/DD systems," in Proc. IEEE GLOBECOM Workshops, Houston, TX, USA, Dec. 5-9, 2011, pp. 782-786.

[19] S. D. Dissanayake and J. Armstrong, "Comparison of ACO-OFDM, DCO-OFDM and ADO-OFDM in IM/DD systems," J. Lightw. Technol., vol. 31, no. 7, pp. 1063-1072, Apr. 2013.

[20] N. Wu and Y. Bar-Ness, "A novel power-efficient scheme asymmetrically and symmetrically clipping optical (ASCO)-OFDM for IM/DD optical systems," EURASIP J. Adv. Signal Process., vol. 2015, no. 3, pp. 1-10, Jan. 2015.

[21] S. C. J. Lee, S. Randel, F. Breyer, and A. M. J. Koonen, "PAM-DMT for intensity-modulated and direct-detection optical communication systems," IEEE Photon. Technol. Lett., vol. 21, no. 23, pp. 1749-1751, Dec. 2009.

[22] A. Weiss, A. Yeredor and M. Shtaif, "Iterative symbol recovery for power-efficient DC-biased optical OFDM systems," J. Lightw. Technol., vol. 34, no. 9, pp. 2331-2338, May 2016.

[23] X. Ling, J. Wang, X. Liang, Z. Ding and C. Zhao, "Offset and power optimization for DCO-OFDM in visible light communication systems," IEEE Trans. Signal Process., vol. 64, no. 2, pp. 349-363, Jan. 2016.

[24] M. Zhang and Z. Zhang, "An optimum DC-biasing for DCO-OFDM system," IEEE Commun. Lett., vol. 18, no. 8, pp. 1351-1354, Aug. 2014.

[25] L. Chen, B. Krongold, and J. Evans, "Theoretical characterization of nonlinear clipping effects in IM/DD optical OFDM systems," IEEE Trans. Commun., vol. 60, no. 8, pp. 2304-2312, Aug. 2012.

[26] Q. Wang, C. Qian, X. Guo, Z. Wang, D. Cunningham, and I. White, "Layered ACO-OFDM for intensity-modulated direct-detection optical wireless transmission," Opt. Express, vol. 23, no. 9, pp. 12382-12393, May 2015.

[27] J. Wang, Y. Xu, X. Ling, R. Zhang, Z. Ding, and C. Zhao, "PAPR analysis for OFDM visible light communication," Opt. Exp., vol. 24, no. 24, pp. 27457-27474, Nov. 2016.

[28] S. Dimitrov, S. Sinanovic, and H. Haas, "Clipping noise in OFDM-based optical wireless communication systems," IEEE Trans. Commun., vol. 60, no. 4, pp. 1072-1081, Apr. 2012.

[29] S. H. Han and J. H. Lee, "An overview of peak-to-average power ratio reduction techniques for multicarrier transmission," IEEE Wireless Commun., vol. 12, no. 2, pp. 56-65, Apr. 2005.

[30] J. Zhou, and Y. Qiao, "Low-PAPR asymmetrically clipped optical OFDM for intensity-modulation/direct-detection systems," IEEE Photon. J., vol. 7, no. 3, pp. 1-8, May 2015.

[31] A. V. Oppenheim, R. W. Schafer, and J. R. Buck, Discrete-Time Signal Processing, 2nd ed. Englewood Cliffs, NJ: Prentice Hall, 1999.
[32] R. Bai, R. Jiang, T. Mao, W. Lei and Z. Wang, "Iterative receiver for ADO-OFDM with near-optimal optical power allocation," Optics Commun., vol. 387, pp. 350-356, Mar. 2017.

[33] H. Yu, M. Chen and G. Wei, "Distribution of PAR in DMT systems," Electron. Lett., vol. 39, no. 10, pp. 799-801, May 2003.

Ruowen Bai received his B.E. degree from Nankai University, Tianjin, China, in 2015. He is currently working toward his M.E. degree at Tsinghua National Laboratory for Information Science and Technology (TNlist), Department of Electronic Engineering, Tsinghua University. His research interest includes visible light communications.

Qi Wang (S'15-M'16) received the B.E. degree and the Ph.D. degree (with highest honor) in electronic engineering from Tsinghua University, Beijing, China, in 2011 and 2016, respectively. From 2014 to 2015, he was a Visiting Scholar with the Electrical Engineering Division, Centre for Photonic Systems, Department of Engineering, University of Cambridge. Since 2016, he has been a Research Fellow with Southampton Wireless Group at University of Southampton. He has authored over 20 IEEE/OSA journal papers and several conference papers. He serves as an Associate Editor for IEEE Access and a TPC member for many IEEE conferences, including Globecom, GlobalSIP, CSNDSP, and IWCMC. His research interests include modulation and signal processing for wireless communication and visible light communication. $\mathrm{He}$ was a recipient of the Excellent Doctoral Dissertation of Chinese Institute of Electronics (CIE), Outstanding Ph.D. Graduate of Tsinghua University, Excellent Doctoral Dissertation of Tsinghua University, National Scholarship, and the Academic Star of Electronic Engineering Department in Tsinghua University.

Zhaocheng Wang (M'09-SM'11) received the B.S., M.S., and Ph.D. degrees from Tsinghua University, Beijing, China, in 1991, 1993, and 1996, respectively. From 1996 to 1997, he was a Postdoctoral Fellow with Nanyang Technological University, Singapore. From 1997 to 1999, he was with OKI Techno Centre (Singapore) Pte. Ltd., Singapore, where he was first a Research Engineer and later became a Senior Engineer. From 1999 to 2009, he was with Sony Deutschland $\mathrm{GmbH}$, where he was first a Senior Engineer and later became a Principal Engineer. He is currently a Professor of Electronic Engineering with Tsinghua University and serves as the Director of Broadband Communication Key Laboratory, Tsinghua National Laboratory for Information Science and Technology (TNlist). He has authored or coauthored over 120 journal papers. He is the holder of 34 granted U.S./EU patents. He coauthored two books, one of which, Millimeter Wave Communication Systems, was selected by IEEE Series on Digital and Mobile Communication (Wiley-IEEE Press). His research interests include wireless communications, visible light communications, millimeterwave communications, and digital broadcasting. $\mathrm{He}$ is a Fellow of the Institution of Engineering and Technology. He served as the Associate Editor of IEEE Transactions on Wireless Communications (2011-2015) and the Associate Editor of IEEE Communications Letters (20132016), and has also served as Technical Program Committee Co-Chair of various international conferences. 